\title{
Multiple omics ageing clocks
}

E. Macdonald-Dunlop ${ }^{1 *}$, P.K. Joshi ${ }^{1}$, J.F. Wilson ${ }^{1,2}$

${ }^{1}$ Centre for Global Health Research, Usher Institute of Population Health Sciences and Informatics, University of Edinburgh, Teviot Place, Edinburgh, UK

${ }^{2}$ MRC Human Genetics Unit, Institute of Genetics and Molecular Medicine, University of Edinburgh, Western General Hospital, Crewe Road, Edinburgh, UK

*e-mail:e.macdonald.dunlop@ed.ac.uk

Key words: ageing, omics, penalized regression, biological age, healthy ageing, multi-omics

Biological age, a measure of deterioration and ageing that is distinct from chronological age has been found to predict disease and mortality [1]. The first published measure of biological age was Hannum's epigenetic clock. Hannum's clock took the form of an elastic net regression model predicting age, built from whole blood $\mathrm{CpG}$ methylation data, the ratio of this predicted age to chronological age was used to measure apparent methylomic ageing rate (AMAR) [2]. Hannum's work was extended by Horvath using the same methodology, but a larger number of: $\mathrm{CpG}$ markers, individuals and tissue types [3].

Since the publication of these landmark epigenetic clocks, ageing clocks have been built using telomere length [4], facial morphology [5], metabolomics [1], glycomics [6] and proteomics [7]. Each of these ageing clocks have shown that chronological age compared to biological age can be used as an indicator of health outlook. These models have the potential to inform health and lifestyle advice in order to improve individuals' health [7]. Here we test replication of published ageing clocks using approximately 1,000 individuals from the cross-sectional population cohort ORCADES, that are highly annotated with 877 phenotypes spanning glycomics, lipidomics, metabolomics and proteomics.

\section{References}

1. Fischer K. et al. (2014) Biomarker profiling by nuclear magnetic resonance spectroscopy for the prediction of all-cause mortality: An observational study of 17,345 persons. Plos Medicine. 11.

2. Hannum G. et al. (2013) Genome-wide methylation profiles reveal quantitative views of human aging rates. Mol. Cell. 49:359-367.

3. Horvath S. (2013) DNA methylation age of human tissues and cell types. Genome Biology. 14.

4. Zhang W.G. et al. (2014) Select aging biomarkers based on telomere length and chronological age to build a biological age equation. Age. 36:1201-1211.

5. Chen W.Y. et al. (2015) Three-dimensional human facial morphologies as robust aging markers. Cell Research. 25:574-587.

6. Kristic J. et al. (2014) Glycans are a novel biomarker of chronological and biological ages. J. Gerontol. Ser. a-Biol. Sci. Med. Sci. 69:779-789.

7. Enroth S., Enroth S.B., Johansson A., Gyllensten U. (2015) Protein profiling reveals consequences of lifestyle choices on predicted biological aging. Sci. Rep. 5. 